\title{
Equations of State of Matter from Shock Wave Experiments
}

\author{
Hitoshi TAKeUchi ${ }^{1}$ \\ Department of Geology and Geophysics, University of California, Berkeley \\ Hiroo Kanamori ${ }^{1}$ \\ Institute of Geological Sciences, California Institute of Technology, Pasadena
}

\begin{abstract}
An interpretation of the shock wave Hugoniot relation is made by using the Mie-Grüneisen equation of state. Isothermal pressure-volume curves for various metals are calculated from the data of McQueen and Marsh and Al'tshuler et al. A linear relation between shock wave velocity $u_{a}$ and particle velocity $u_{p}$ is assumed, and the numerical integration is extended beyond the range of the experiments. Independent calculations are made by using either the Slater or the Dugdale-MacDonald formula. Results thus obtained do not differ appreciably. The result for iron is in good agreement with that obtained by Al'tshuler et al., who used an experimentally determined value of the Grüneisen ratio. The equations of state thus calculated are well approximated by the Murnaghan-Birch equation of state with the second-order coefficient $\xi$ varying from $-1 / 2$ to $1 / 2$. The use of the TFD model is not valid below $100 \mathrm{Mb}$. However, inspection of the results gives the impression that for some metal the $P$-p curve will approach the TFD curve at pressures higher than $100 \mathrm{Mb}$. The density and incompressibility of the earth's core are compared with those of iron. The general conclusion is that the density of the earth's core is about 1 to $1.5 \mathrm{~g} / \mathrm{cm}^{3}$ less than the density of iron at corresponding pressure and temperature. The difference in incompressibility is very small. Bullen's incompressibility-pressure hypothesis is tested on the basis of the result obtained here. The variation of the values of incompressibility among the metals studied here is too large even at the pressure of $4 \mathrm{Mb}$ to support the hypothesis. A detailed inspection of the result for the incompressibility obtained here, however, implies that it is still difficult to avoid the conclusion arrived at from the incompressibility-pressure hypothesis that the inner core is solid.
\end{abstract}

\section{INTRODUCTION}

Shock wave studies have provided valuable information for the study of the internal constitution of the earth. Rice et al. [1958] determined the equition of state for a number of materials to pressures of up to $500 \mathrm{~kb}$. The agreement of their results with the static measurements of Bridgman could be regarded as a proof of the soundness of the basic assumptions. The pressure range was successively extended to $2 \mathrm{Mb}$ by $M c Q u e e n$ and Marsh [1960]. Al'tshuler et al. $[1958 a, b, 1960]$ studied the shock compression of various metals at still higher pressures (up to 4 to $5 \mathrm{Mb}$ ). As the shock wave experiments give the relationship between pressure $P$ and volume $v$ under the shock condition, it is necessary to introduce thermodynamical considerations in order to interpret them in terms of isothermal or adiabatic $P-v$ relations.

${ }^{1}$ On leave from Geophysical Institute, University of Tokyo.
In the works of Rice et al. [1958] and $M c$ Queen and Marsh [1960] the Mie-Grüneisen equation of state and the Dugdale-MacDonald relation for the volume dependence of the Grüneisen ratio were introduced for the interpretation of the Hugoniot $P-v$ relation. On the other hand, Al'tshuler et al. performed an elaborate experiment using a reduced density sample to determine the Grüneisen ratio at high pressures. Using this and the Mie-Grüneisen equation of state, they determined the equation of state of iron. For other elements, however, equations of state were not given for lack of an experimentally determined Grüneisen ratio at high pressures.

In this paper we attempt to formulate as systematically as possible the method of interpreting the Hugoniot $P-v$ relation and then to make the Hugoniot data so far determined readily available for use in various geophysical problems. We also discuss the equation of state in relation to the Murnaghan-Birch equation of 
state for moderately high pressure and the Thomas-Fermi-Dirac equation of state for extremely high pressures. The variation with pressure of incompressibility $k$, Debye's temperature $\theta$, and the Grüneisen ratio $\gamma$ are also calculated. The method is also extended to the calculation of isothermal pressure-density relations for elevated temperatures. All these data are used in discussing problems of basic geophysical interest, such as Bullen's incompressibility-pressure hypothesis and the distribution of density and incompressibility in the earth's core.

\section{MeTHOD OF INTERPRETATION}

Shock wave experiments determine the shock wave velocity $u_{a}$ and particle velocity $u_{p}$ in the sample. Using the laws of conservation of mass, momentum, and energy, we can derive the following relations [Rice et al., 1958]:

$$
\begin{array}{r}
u_{s}=v_{H 0}\left[\left(P_{H}-P_{H 0}\right) /\left(v_{H 0}-v\right)\right]^{1 / 2} \\
u_{p}=\left[\left(P_{H}-P_{H 0}\right)\left(v_{H 0}-v\right)\right]^{1 / 2} \\
E_{H}=E_{H 0}+\frac{1}{2}\left(P_{H}-P_{H 0}\right)\left(v_{H 0}-v\right)
\end{array}
$$

where $v, P_{I I}$, and $E_{I}$ denote the specific volume, pressure, and internal energy of the sample under shock compression. $v_{H 0}, P_{H 10}$, and $E_{H 0}$ are the quantities corresponding to the state before the passage of the shock front. A set of $u_{e}$ and $u_{p}$ values determined experimentally under various shock pressures provides a Hugoniot $P-v$ relation through (1) and (2). Generally, $u$, and $u_{p}$ for a single material can conveniently be expressed by the relation

$$
u_{\mathrm{s}}=C_{0}+\lambda u_{p}
$$

where $C_{0}$ and $\lambda$ are constants characteristic of the material considered. Although this relation does not seem to have any theoretical basis, it fits the data remarkably well [Alder, 1963]. By introducing the relation, we can express the experimental results simply by the two parameters $C_{0}$ and $\lambda$, which in turn give an explicit Hugoniot relation through (1), (2), and (4) as

$$
P_{H}=P_{H 0}+\frac{C_{0}{ }^{2}\left(v_{H 0}-v\right)}{\left[v_{H 0}-\lambda\left(v_{H 0}-v\right)\right]^{2}}
$$

Since $P_{H}$ denotes the pressure under the shock condition with elevated temperature, it is necessary to remove from it the temperature effect to obtain the $0^{\circ} \mathrm{K}$ isothermal equation of state.
For this purpose the Mie-Grüneisen equation o state is introduced:

$$
P_{H}-P_{K}=\gamma\left(E_{H}-E_{K}\right) / v
$$

where $\gamma$ is Grüneisen's ratio and $P_{E}$ is the pres sure necessary for compressing at $0^{\circ} \mathrm{K}$ a mate. rial to a state having the same specific volum $v$ as that under the shock compression. $E_{\mathrm{r}}$ is the internal energy for the $0^{\circ} \mathrm{K}$ isothermal com. pression and is related to $P_{K}$ by

$$
P_{K}=-\left(\partial E_{K} / \partial v\right)_{T}
$$

Putting (3) into (6) leads to

$$
\gamma=\frac{v\left(P_{t I}-P_{K}\right)}{\frac{1}{2}\left(P_{H}-P_{H 0}\right)\left(v_{H 0}-v\right)+E_{H 0}-E_{K}} \text { (8) }
$$

The volume dependence of Grüneisen's ratio was given by Slater and later by Dugdale and MacDonald [see Rice et al., 1958]. Both formulas can be written as

$$
-2(\gamma+\beta) / v=\frac{d^{2}\left(P_{K} v^{\alpha}\right)}{d v^{2}} / \frac{d\left(P_{K} v^{\alpha}\right)}{d v}
$$

where $\alpha$ and $\beta$ are constants. In Slater's formula $\alpha=0$ and $\beta=2 / 3$, and in Dugdale and MacDonald's formula $\alpha=2 / 3$ and $\beta=1 / 3$. Equations 7,8 , and 9 are the basic equations determining the $0^{\circ} \mathrm{K}$ isothermal $P-v$ relation of the material. In integrating these, we used the following procedures. We let $v_{K O}$ be the specific volume of the material at $0^{\circ} \mathrm{K}$ and zero pressure. Introducing new variables $x$ and $q_{s}$, defined by

and

$$
x=v_{\kappa 0} / v-1
$$

$$
\partial P_{K} / \partial x=q_{K}
$$

we can rewrite (7), (8), and (9) as

$$
\begin{aligned}
& \quad \partial E_{K} / \partial x=v_{K 0} P_{K} /(1+x)^{2} \\
& \frac{\partial q_{K}}{\partial x}=\frac{2}{1+x}\left(\gamma-\frac{1}{3}\right) q_{K} \quad \text { (Slater) } \\
& \frac{\partial q_{K}}{\partial x}=\frac{2 \gamma}{1+x} g_{K}-\frac{4}{3} \frac{1}{(1+x)^{2}} \\
& \cdot\left(\gamma+\frac{1}{6}\right) P_{K} \quad \text { (Dugdale-MacDonald) } \\
& \text { where }
\end{aligned}
$$

$$
\begin{gathered}
\gamma=\left(P_{H}-P_{K}\right) /\left\{\frac{1}{2} P_{H}\left[\frac{v_{H 0}}{v_{K 0}}(1+x)-1\right]\right. \\
\left.+\frac{(1+x)}{v_{K 0}}\left[E_{E 0}-E_{K}\right]\right\}
\end{gathered}
$$


Equations 11, 12, 13 (or 13a), and 14 form a set of differential equations to determine $E_{K}, P_{K}$, $q_{x}$, and $\gamma$. In these equations, $P_{\boldsymbol{x}}$ is experimentally determined as in (5) and can be explicitly written by using the new variable $x$ as

$$
\begin{aligned}
& P_{H}=\frac{C_{0}^{2}}{v_{H 0}}(1+x) \\
& \cdot \frac{x+\left(1-v_{K 0} / v_{H 0}\right)}{\left[x(1-\lambda)+1-\lambda\left(1-v_{K 0} / v_{H 0}\right)\right]^{2}}
\end{aligned}
$$

$E_{g_{0}}$ is the internal energy in the normal state $\left(20^{\circ} \mathrm{C}, 1 \mathrm{~atm}\right)$ and can be calculated by Debye's formula

$$
E_{H 0}=\frac{3 K T N_{0}}{w} u\left(\frac{\Theta}{T}\right)
$$

where $K, N_{0}, w$, and $\Theta$ are Boltzmann's constant, Avogadro's number, atomic weight, and Debye's temperature, respectively. The function $u$ is given by

$$
u(x)=\frac{3}{x^{3}} \int_{0}^{x} \frac{\xi^{3}}{e^{\xi}-1} d \xi
$$

which is tabulated in a number of textbooks [see, e.g., Knopoff, 1963].

The value of $v_{\pi_{0}}$ is not known at first but can be estimated in the following manner. Because the difference between $v_{z r}$ and $v_{T 0}$ is due to thermal expansion, $v_{x o}$ can be written as

$$
v_{K 0}=v_{H 0}\left(1-\int_{0}^{293} \alpha d T\right)
$$

where $\alpha$ is the thermal expansion coefficient. Introducing the thermodynamical relation

$$
\alpha=\gamma C / k v
$$

where $C$ and $k$ denote the specific heat at constant pressure and the adiabatic incompressibility, and making use of the fact that $\gamma, k$, and $v$ all vary slowly with temperature, we can write (18) as

$$
\begin{array}{r}
v_{R 0} \approx v_{H 0}\left(1-\frac{\gamma_{H 0}}{k_{B 0} v_{H 0}} \int_{0}^{293} C d T\right) \\
\approx v_{B 0}\left(1-\frac{\gamma_{H 0} E_{H 0}}{l_{H 0} v_{B 0}}\right)
\end{array}
$$

In the last equation in (20), we neglect a small difference between the specific heat at constant pressure and constant volume. For all the ele- ments except iron, we used the thermodynamically determined values of $\gamma$ listed by McQueen and Marsh [1960]. For iron we used the relation

$$
\gamma_{H 0}=2 \lambda-1
$$

The value thus obtained is 2.16 , which is close to the experimentally determined value 1.95 given by Al'tshuler et al. [1958]. The values of $k_{z 0}$ are estimated for the foot of the Hugoniot curve, namely,

$$
k_{H O}=-v_{I T 0}\left(\frac{\partial P_{H}}{\partial v}\right)_{v H 0}=\frac{C_{0}{ }^{2}}{v_{H O}}
$$

Given all these values $v_{\bar{K} 0}$ can readily be estimated.

The initial conditions necessary for the integration of (11), (12), and (13) are

$$
\begin{gathered}
P_{K}=0 \quad E_{K}=0 \text { at } x=0 \\
q_{K}=C_{0}{ }^{2} / v_{H 0}
\end{gathered}
$$

The first and second conditions are evident, but the third condition which gives the initial slope of $P_{K}$ might require explanation. Although there is no theoretical estimation of the initial slope of the $P_{x}$ curve, the initial slope of the Hugoniot $P_{n}$ probably approximates it closely, because at the foot of the Hugoniot the temperature is not greatly raised and we can neglect a small difference between the isothermal $\partial P_{K} / \partial v$ and adiabatic $\partial P_{H} / \partial v$ slope. The slope at the foot of the Hugoniot is, as shown in (22), $C_{0}^{2} / V_{y 0}$, which is now used as the initial value of $q_{\mathrm{k}}$. We can avoid the estimation of $v_{K 0}$ as in (18) by starting our numerical integration from $v=v_{\text {H0 }}$. In this case, however, we must estimate $P_{K}$ and $E_{x}$ at $v=v_{H 0}$, which are not zero, and in estimating them we must use approximations such as are used in (18)-(20).

We numerically integrate for $\mathrm{Fe}, \mathrm{Cu}, \mathrm{Zn}, \mathrm{Ag}$, $\mathrm{Cd}, \mathrm{Au}$, and $\mathrm{Pb}$ using the data of Al'tshuler et al. [1958] and for $\mathrm{Ag}, \mathrm{Au}, \mathrm{Cd}, \mathrm{Co}, \mathrm{Cr}, \mathrm{Cu}, \mathrm{Mo}$, $\mathrm{Ni}, \mathrm{Pb}, \mathrm{Sn}$ (gray), $\mathrm{Sn}$ (white), $\mathrm{Th}, \mathrm{Ti}, \mathrm{Tl}, \mathrm{V}, \mathrm{W}$, and $\mathrm{Zn}$ using the data of McQueen and Marsh [1960]. The integrations for each metal are made twice, first with the Slater formula and then with the Dugdale-MacDonald formula. All the constants in the calculations are summarized in Table 1.

In Table 2 to 4, part of the results obtained by using the data of Al'tshuler et al. are given. 
TABLE 1. Summary of Constants Used in the Calculation

\begin{tabular}{|c|c|c|c|c|c|c|c|c|}
\hline & $Z$ & $w$ & $\gamma_{H 0^{*}}{ }^{*}$ & $\begin{array}{c}C_{0}, \dagger \\
\mathrm{km} / \mathrm{sec}\end{array}$ & $\lambda \dagger$ & $\begin{array}{c}E_{H 0}, \\
10^{8} \mathrm{ergs}\end{array}$ & $\begin{array}{c}v_{H 0, \dagger} \dagger \\
\mathrm{cm}^{3} / \mathrm{g}\end{array}$ & $\begin{array}{c}\Theta_{0,} \ddagger \\
{ }^{\circ} \mathrm{K}\end{array}$ \\
\hline $\mathrm{Ag}$ & 47 & 107.88 & 2.55 & 3.3 & 1.54 & 5.03 & 0.09533 & 225 \\
\hline AlI & 79 & 197.0 & 3.29 & 3.15 & 1.47 & 2.98 & 0.0518 & 165 \\
\hline $\mathrm{Cd}$ & 48 & 112.41 & 2.32 & 2.65 & 1.48 & 4.35 & 0.116 & 300 \\
\hline $\mathrm{Cu}$ & 29 & 63.54 & 2.00 & 3.9 & 1.46 & 6.24 & 0.112 & 445 \\
\hline $\mathrm{Fe}$ & 26 & 55.85 & 2.16 & 3.8 & 1.58 & 6.88 & 0.127 & 467 \\
\hline $\mathbf{P b}$ & 82 & 207.21 & 2.77 & 2.3 & 1.27 & 3.12 & 0.0882 & 94.3 \\
\hline $\mathrm{Zn}$ & 30 & 65.38 & 2.45 & 3.2 & 1.45 & 7.38 & 0.140 & 308 \\
\hline $\mathrm{Ag}$ & 47 & 107.88 & 2.55 & 3.243 & 1.586 & 5.09 & 0.0953 & 215 \\
\hline $\mathrm{Au}$ & 79 & 197.00 & 3.29 & 3.075 & 1.560 & 2.96 & 0.0520 & 170 \\
\hline $\mathrm{Cd}$ & 48 & 112.41 & 2.32 & 2.443 & 1.671 & 5.56 & 0.1157 & 120 \\
\hline Co & 27 & 58.94 & 1.97 & 4.748 & 1.330 & 7.34 & 0.1134 & 385 \\
\hline Cr & 24 & 52.01 & 1.19 & 5.217 & 1.465 & 7.47 & 0.1408 & 460 \\
\hline $\mathrm{Cu}$ & 29 & 63.54 & 2.00 & 3.958 & 1.497 & 7.52 & 0.1124 & 315 \\
\hline Mo & 42 & 95.95 & 1.52 & 5.157 & 1.238 & 4.54 & 0.0980 & 380 \\
\hline $\mathrm{Ni}$ & 28 & 58.71 & 1.83 & 4.646 & 1.445 & 7.48 & 0.1129 & 375 \\
\hline $\mathrm{Pb}$ & 82 & 207.21 & 2.77 & 2.028 & 1.517 & 3.15 & 0.0882 & 88 \\
\hline \multirow[t]{2}{*}{$\mathrm{Sn}$} & 50 & 118.70 & 2.11 & 2.640 & 1.476 & 4.35 & 0.1374 & 260 \\
\hline & & & & & & 4.92 & & 170 \\
\hline Th & 90 & 232.05 & 1.26 & 2.132 & 1.278 & 2.77 & 0.0856 & 100 \\
\hline $\mathrm{Ti}$ & 22 & 47.90 & 1.09 & 4.779 & 1.089 & 9.08 & 0.2217 & 380 \\
\hline $\mathrm{Tl}$ & 81 & 204.39 & 2.25 & 1.859 & 1.515 & 3.16 & 0.0845 & 96 \\
\hline$\vec{V}$ & 23 & 50.95 & 1.29 & 5.108 & 1.210 & 8.43 & 0.1639 & 390 \\
\hline W & 74 & 183.86 & 1.54 & 4.005 & 1.268 & 2.02 & 0.0522 & 310 \\
\hline $\mathrm{Zn}_{\mathrm{n}}$ & 30 & 65.38 & 2.45 & 3.050 & 1.559 & 8.19 & 0.1400 & 234 \\
\hline
\end{tabular}

* From McQueen and Marsh [1960].

$\dagger$ For the top set, from $A l^{\prime} t s h u l e r$ et al. [1958]; for the bottom set, from McQueen and Marsh [1960].

$\ddagger$ For the top set, from Kittel [1956]; for the bottom set, from Launay [1956].

TABLE 2. Summary of Calculation for Silver

\begin{tabular}{|c|c|c|c|c|c|c|c|c|c|c|c|c|c|}
\hline \multirow{2}{*}{$\frac{V_{K v}}{V}$} & \multicolumn{7}{|c|}{ Dugdale-MacDonald } & \multicolumn{6}{|c|}{ Slater } \\
\hline & $\begin{array}{l}P_{I J} \\
\mathrm{Mb}\end{array}$ & $\begin{array}{l}P_{K} \\
\mathrm{Mb}\end{array}$ & $\begin{array}{l}k, \\
\text { Mb }\end{array}$ & $\boldsymbol{\gamma}$ & $\begin{array}{c}E_{K} \\
10^{10} \text { ergs }\end{array}$ & $\begin{array}{l}\Theta, \\
{ }^{\circ} \mathbf{K}\end{array}$ & $\begin{array}{c}T_{H}, \\
{ }^{\circ} \mathrm{K}\end{array}$ & $\begin{array}{l}P_{K} \\
\mathrm{Mb}\end{array}$ & $\begin{array}{l}k, \\
\mathrm{Mb}\end{array}$ & $\gamma$ & $\begin{array}{c}E_{K}, \\
10^{10} \text { ergs }\end{array}$ & $\stackrel{\Theta}{,}_{{ }_{2}} \mathbf{K}$ & $\begin{array}{l}T_{I I}, \\
{ }^{0} \mathbf{K}\end{array}$ \\
\hline 1.00 & 0.01 & 0 & 1.14 & 2.57 & 0 & 225 & 300 & 0 & 1.14 & 2.57 & 0 & 225 & 300 \\
\hline 1.10 & 0.16 & 0.15 & 1.96 & 2.00 & 0.06 & 283 & 430 & 0.14 & 1.91 & 2.31 & 0.06 & 287 & 440 \\
\hline 1.20 & 0.38 & 0.35 & 2.81 & 1.54 & 0.23 & 327 & 740 & 0.35 & 2.79 & 1.80 & 0.22 & 341 & 780 \\
\hline 1.30 & 0.69 & 0.62 & 3.82 & 1.66 & 0.52 & 372 & 1,600 & 0.61 & 3.81 & 1.76 & 0.51 & 393 & 1,600 \\
\hline 1.40 & 1.13 & 0.94 & 5.12 & 1.70 & 0.91 & 422 & 3,300 & 0.94 & 5.06 & 1.75 & 0.90 & 448 & 3,500 \\
\hline 1.50 & 1.73 & 1.35 & 6.69 & 1.67 & 1.42 & 474 & 6,400 & 1.34 & 6.58 & 1.71 & 1.41 & 505 & 6,600 \\
\hline 1.60 & 2.59 & 1.84 & 8.54 & 1.62 & 2.04 & 527 & 12,000 & 1.81 & 8.35 & 1.66 & 2.02 & 563 & 12,000 \\
\hline 1.70 & 3.80 & 2.42 & 10.67 & 1.57 & 2.78 & 580 & 22,000 & 2.38 & 10.39 & 1.60 & 2.74 & 621 & 21,000 \\
\hline 1.80 & 5.54 & 3.10 & 13.09 & 1.53 & 3.62 & 634 & 35,000 & 3.04 & 12.08 & 1.56 & 3.57 & 680 & 36,000 \\
\hline 1.90 & 8.07 & 3.88 & 15.80 & 1.49 & 4.58 & 688 & 57,000 & 3.79 & 15.25 & 1.51 & 4.51 & 739 & 62,000 \\
\hline 2.00 & 11.88 & 4.76 & 18.81 & 1.45 & 5.64 & 742 & & 4.65 & 18.08 & 1.47 & 5.55 & 797 & \\
\hline 2.10 & 17.79 & 5.76 & 22.12 & 1.41 & 6.82 & 795 & & 5.60 & 21.16 & 1.43 & 6.70 & 856 & \\
\hline 2.20 & 27.43 & 6.87 & 25.72 & 1.38 & 8.10 & 849 & & 6.66 & 24.50 & 1.39 & 7.94 & 914 & \\
\hline 2.30 & 44.21 & 8.10 & 29.60 & 1.33 & 9.49 & 901 & & 7.83 & 28.07 & 1.34 & 9.29 & 971 & \\
\hline 2.40 & 76.31 & 9.44 & 33.75 & 1.29 & 10.98 & 953 & & 9.10 & 31.86 & 1.30 & 10.73 & 1027 & \\
\hline 2.50 & 147.3 & 10.91 & 38.15 & 1.25 & 12.58 & 1004 & & 10.48 & 35.84 & 1.25 & 12.26 & 1082 & \\
\hline 2.60 & 348.3 & 12.50 & 42.76 & 1.20 & 14.27 & 1053 & & 11.97 & 39.97 & 1.20 & 13.89 & 1135 & \\
\hline 2.70 & 1,320 & 14.20 & 47.55 & 1.15 & 16.06 & 1101 & & 13.56 & 44.22 & 1.15 & 15.59 & 1186 & \\
\hline 2.80 & 67,766 & 16.02 & 52.50 & 1.09 & 17.94 & 1147 & & 15.24 & 48.56 & 1.09 & 17.39 & 1236 & \\
\hline
\end{tabular}


TABLE 3. Summary of Calculation for Iron

\begin{tabular}{|c|c|c|c|c|c|c|c|c|c|c|c|c|c|}
\hline \multirow[b]{2}{*}{$\frac{V_{K \mathbf{0}}}{V}$} & \multicolumn{7}{|c|}{ Dugdale-MacDonald } & \multicolumn{6}{|c|}{ Slater } \\
\hline & $\begin{array}{l}P_{H} \\
\mathrm{Mb}\end{array}$ & $\begin{array}{l}P_{K}, \\
\mathrm{Mb}\end{array}$ & $\begin{array}{c}k, \\
\mathrm{Mb}\end{array}$ & $\boldsymbol{\gamma}$ & $\begin{array}{c}E_{K} \\
10^{10} \mathrm{ergs}\end{array}$ & ${ }^{\Theta} \mathrm{G}$, & $\stackrel{T_{H},}{{ }^{\circ} \mathrm{K}}$ & $\begin{array}{l}P_{K}, \\
\mathrm{Mb}\end{array}$ & $\stackrel{k,}{\mathrm{Mb}}$ & $\gamma$ & $\begin{array}{c}E_{K}, \\
10^{10} \mathrm{ergs}\end{array}$ & $\begin{array}{l}\Theta, \\
{ }^{\circ} \mathrm{K}\end{array}$ & $\begin{array}{l}T_{H}, \\
{ }^{\circ} \mathrm{K}\end{array}$ \\
\hline 00 & 0.01 & 0 & 1.14 & 2.18 & 0 & 467 & 290 & 0 & 1.14 & 2.18 & 0 & 467 & 290 \\
\hline 1.10 & 0.16 & 0.14 & 1.90 & 2.20 & 0.07 & 580 & 400 & 0.14 & 1.86 & 2.51 & 0.07 & 587 & 400 \\
\hline 1.20 & 0.38 & 0.35 & 2.86 & 1.80 & 0.30 & 688 & 670 & 0.34 & 2.84 & 2.04 & 0.29 & 717 & 690 \\
\hline 1.30 & 0.70 & 0.62 & 3.98 & 1.74 & 0.68 & 792 & 1,300 & 0.61 & 3.96 & 1.83 & 0.67 & 835 & 1,300 \\
\hline 1.40 & 1.16 & 0.96 & 5.37 & 1.73 & 1.22 & 900 & 2,600 & 0.95 & 5.31 & 1.78 & 1.20 & 954 & 2,700 \\
\hline 1.50 & 1.81 & 1.39 & 7.06 & 1.71 & 1.92 & 1014 & 5,100 & 1.37 & 6.93 & 1.75 & 1.89 & 1077 & 5,100 \\
\hline 1.60 & 2.75 & 1.91 & 9.06 & 1.67 & 2.77 & 1130 & 9,400 & 1.88 & 8.85 & 1.71 & 2.74 & 1205 & 10,000 \\
\hline 1.70 & 4.11 & 2.52 & 11.40 & 1.63 & 3.79 & 1249 & 16,000 & 2.48 & 11.08 & 1.67 & 3.74 & 1334 & 17,000 \\
\hline 1.80 & 6.14 & 3.25 & 14.07 & 1.59 & 4.97 & 1370 & 30,000 & 3.19 & 13.63 & 1.62 & 4.90 & 1466 & 29,000 \\
\hline 1.90 & 9.23 & 4.09 & 17.10 & 1.56 & 6.31 & 1492 & 50,000 & 4.00 & 16.50 & 1.58 & 6.21 & 1598 & 50,000 \\
\hline 2.00 & 14.10 & 5.05 & 20.49 & 1.52 & 7.82 & 1614 & 85,000 & 4.92 & 19.68 & 1.53 & 7.68 & 1731 & 87,000 \\
\hline 2.10 & 22.18 & 6.14 & 24.23 & 1.48 & 9.49 & 1737 & 150,000 & 5.97 & 23.18 & 1.49 & 9.31 & 1863 & 140,000 \\
\hline 2.20 & 36.58 & 7.36 & 28.32 & 1.43 & 11.32 & 1858 & & 7.13 & 26.98 & 1.44 & 11.08 & 1995 & \\
\hline 2.30 & 64.95 & 8.72 & 32.75 & 1.39 & 13.31 & 1978 & & 8.42 & 31.06 & 1.39 & 13.01 & 2124 & \\
\hline 2.40 & 130.5 & 10.21 & 37.49 & 1.34 & 15.46 & 2096 & & 9.83 & 35.39 & 1.34 & 15.08 & 2251 & \\
\hline 2.50 & 332.1 & 11.84 & 42.50 & 1.28 & 17.76 & 2212 & & 11.37 & 39.93 & 1.28 & 17.30 & 2375 & \\
\hline 2.60 & 1,535 & 13.61 & 47.76 & 1.22 & 20.22 & 2323 & & 13.03 & 44.63 & 1.22 & 19.66 & 2495 & \\
\hline 2.70 & 9,859 & 15.51 & 53.20 & 1.16 & 22.82 & 2430 & & 14.80 & 49.45 & 1.16 & 22.14 & 2610 & \\
\hline
\end{tabular}

Other results obtained will be given elsewhere. The $P_{x-\rho}(=1 / v)$ relations for seven metals thus obtained are shown in Figures 1 to 7 .

As is easily seen from (5), $P_{K I}$ becomes infinitely large when $v$ reaches the value $V_{n 0}$ $(\lambda-1) / \lambda$. The physical significance of this is not clear [Alder, 1963], but the function cannot be integrated beyond this limit. As the value of $\lambda$ is around 1.5 for most of the metals, this limit is set at $v \approx 0.3 v_{n 0}$ or $x \approx 2$. In the present study, we assumed that the linear relation (4)

TABLE 4. Comparison of the Result for Iron with the Result Obtained by Al'tshuler et al. [1958]

\begin{tabular}{cccc}
$\frac{V_{0}}{V}$ Al'tshuler et al. & \multicolumn{2}{c}{$\begin{array}{c}P_{\mathbf{K}}, \mathrm{Mb} \\
{[1958]}\end{array}$} & $\begin{array}{c}\text { Dugdale-MacDongld } \\
\text { Slater }\end{array}$ \\
\hline 1.25 & 0.463 & 0.47 & 0.47 \\
1.30 & 0.600 & 0.62 & 0.61 \\
1.35 & 0.761 & 0.78 & 0.77 \\
1.40 & 0.942 & 0.96 & 0.95 \\
1.45 & 1.154 & 1.17 & 1.15 \\
1.50 & 1.358 & 1.39 & 1.37 \\
1.55 & 1.613 & 1.64 & 1.62 \\
1.60 & 1.880 & 1.91 & 1.88 \\
1.65 & 2.174 & 2.20 & 2.17 \\
1.70 & 2.484 & 2.52 & 2.48 \\
1.75 & 2.822 & 2.87 & 2.82 \\
\hline
\end{tabular}

was valid at the high pressures not covered by the experiment, and we integrated up to $v \approx$ ${ }^{\prime}{ }^{\prime}{ }_{0}(\lambda-1) / \lambda$, where the volume was about onethird of the initial value. It should be noted that this is just an extrapolation. The Hugoniot at $4 \mathrm{Mb}$, say, is almost certainly in the melting range, which menns that different $C_{0}$ and $\lambda$ should be used.

\section{Variation of $\gamma, \theta$, and Temperature}

The Grüneisen ratio $\gamma$ hils been determined as a function of volume by using (14). The variation with volume of the Debye temperature $\theta$ can also be determined by integrating the basic formula [see Knopoff, 1963].

$$
\gamma=-(v / \Theta)(d \Theta / d v)
$$

Tables 2 and 3 include the variation of $\gamma$ and $\theta$ thus obtained.

Having determined the values of $P_{K}, \gamma$, and $\theta$ as functions of volume, we can calculate the temperature along the Hugoniot as well as the thermal pressure, pressure necessary to offset the effect of thermal expansion, from which in turn, if necessary, isotherms at arbitrary temperatures can be obtained. The procedure is as follows. Combining the Mie-Grüneisen equation of state with Debye's formula for the thermal 


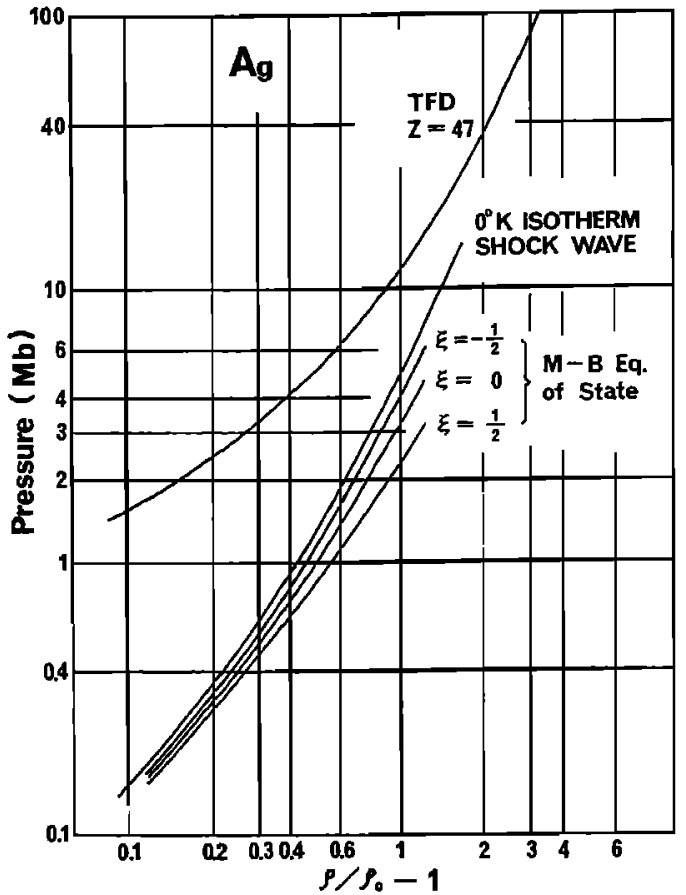

Fig. 1. Isotherms of silver at $0^{\circ} \mathrm{K}$ based on shock wave data, Murnaghan-Birch model and Thomas-Fermi-Dirac model.

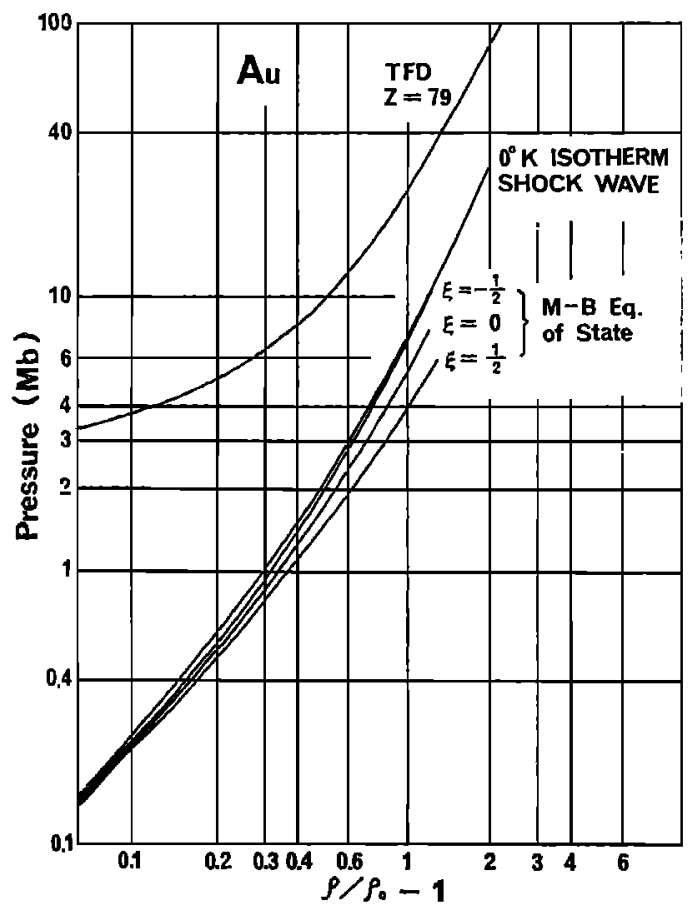

Fig. 2. Isotherms of gold at $0^{\circ} \mathrm{K}$.

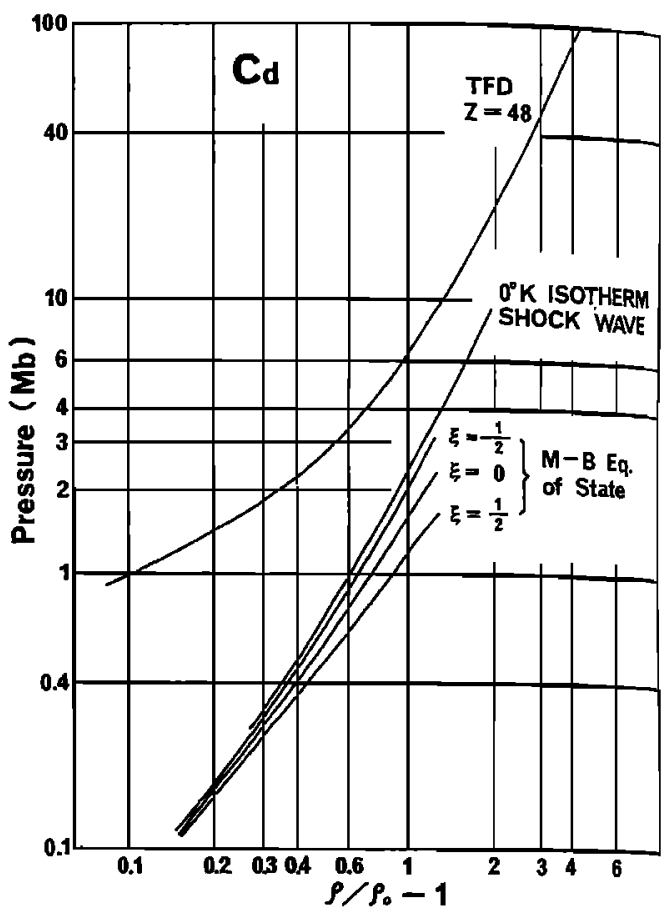

Fig. 3. Isotherms of eadmium at $0^{\circ} \mathrm{K}$.

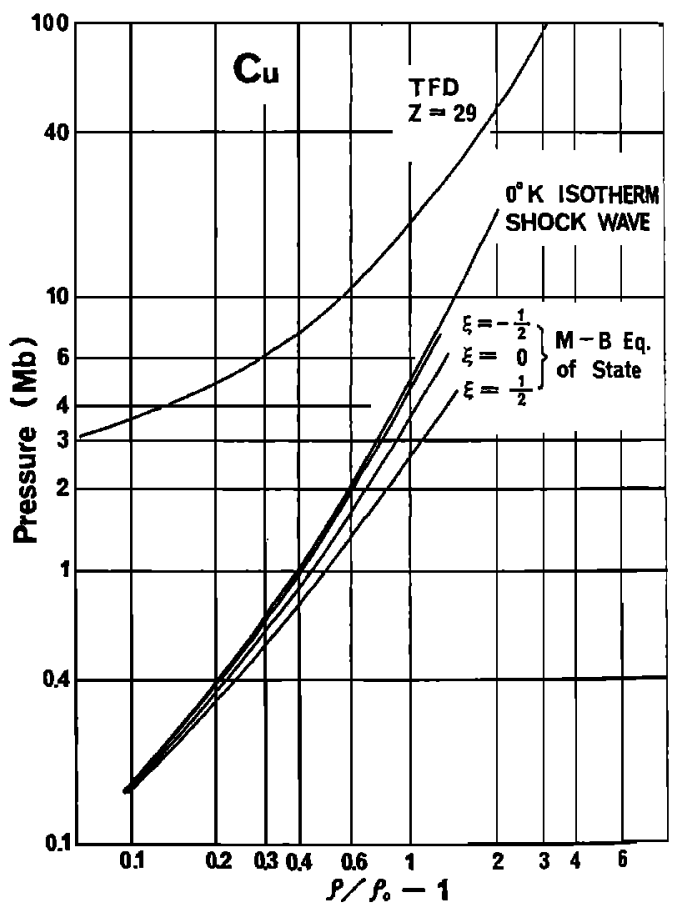

Fig. 4. Isotherms of copper at $0^{\circ} \mathrm{K}$. 


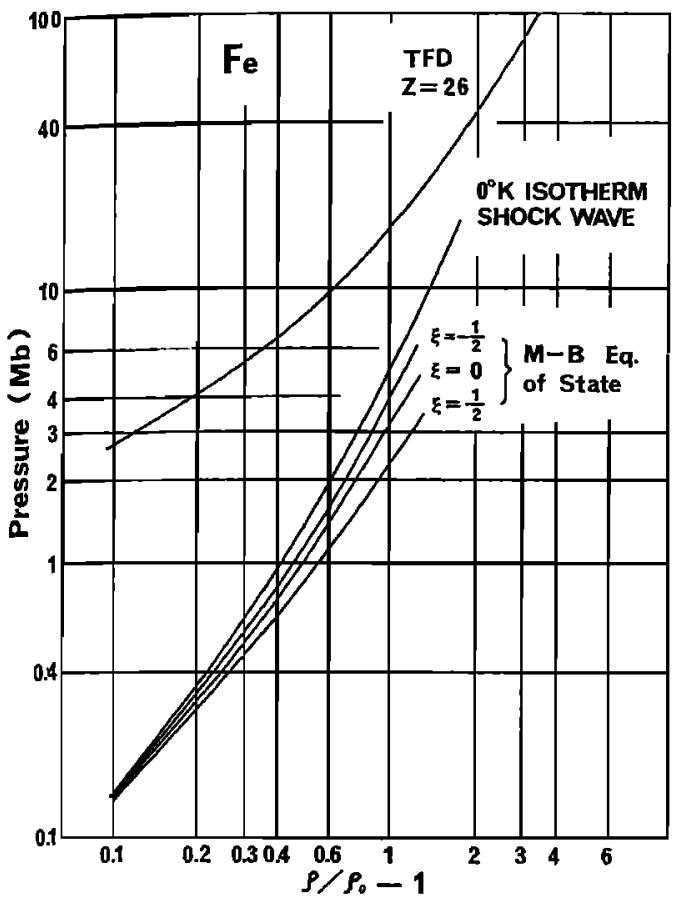

Fig. 5. Isotherms of iron at $0^{\circ} \mathrm{K}$.

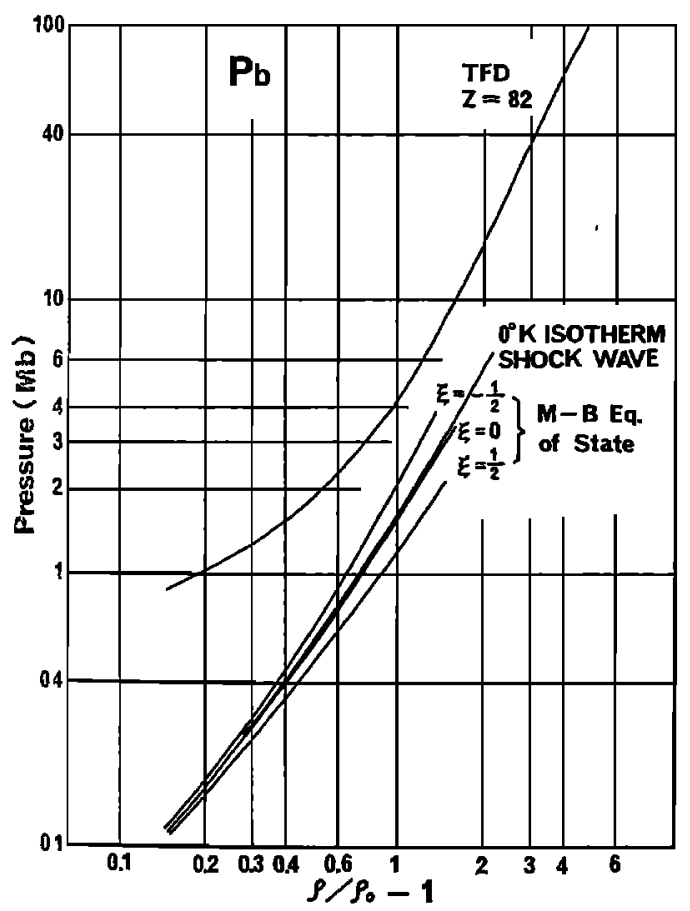

Fig. 6. Isotherms of lead at $0^{\circ} \mathrm{K}$.

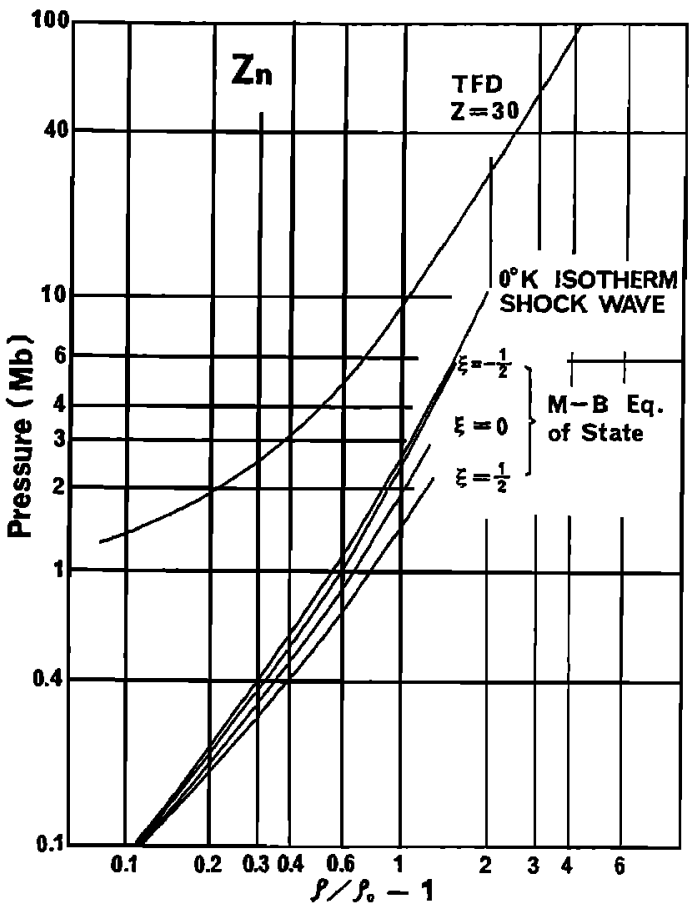

Fig. 7. Isotherms of zinc at $0^{\circ} \mathrm{K}$.

energy, we obtain

$$
P-P_{K}=(\gamma / v) 3 N_{0} K(T / w) u(\Theta / T)
$$

where $P$ is the pressure necessary to compress a material to the state having specific volume at temperature $T$. Since $P_{E}, \gamma$, and $\theta$ are all known as functions of $v$, the isotherm at temperature $T$ can be calculated by adding the right-hand side of (25) to $P_{x}$. Similarly, $T$ along the Hugoniot can be determined by equating $P_{H}$ to $P$ in (25) and solving it for $T$. The values of $T$ thus determined are given in Tables 2, 3, and 4 .

\section{Discussion of the Rmsults}

In Table 4, the result obtained here for iron is compared with that obtained by Al'tshuler et al. [1958] in which the Grüneisen ratio is experimentally determined. The agreement of the present result with that of Al'tshuler et al. is remarkable. No such comparison can be made for other metals because of lack of experimentally determined values of Grüneisen's ratio at high pressures. However, the close agreement found for iron implies the appropriateness of the present method for the interpretation of the 
Hugoniot relations for other metals. Also, the difference between the results based on the Dugdale-MacDonald formula and the Slater formula is negligible. For $\mathrm{Ag}, \mathrm{Au}, \mathrm{Cd}, \mathrm{Cu}, \mathrm{Pb}$, and $\mathrm{Zn}$, the calculation was based on the data of both Al'tshuler et al. and McQueen and Marsh. Although the discrepancy between the results from the two sets of data is small for $\mathrm{Ag}, \mathrm{Au}, \mathrm{Cu}$, and $\mathrm{Zn}$, appreciable differences were observed for $\mathrm{Cd}$ and $\mathrm{Pb}$. This result is not unexpected because the values of $C_{0}$ and $\lambda$, which mainly determine the equation of state, differ considerably (Table 1). For the above $\operatorname{six}, P_{\Sigma}-v$ curves in Figures 1 to 7 are from the data of Al'tshuler et al.

The equations of state at $0^{\circ} \mathrm{K}$ calculated here are compared with the Murnaghan-Birch and Thomas-Fermi-Dirac equations of state. The Murnaghan-Birch equations of state are calculated for $\xi=-1 / 2,0,1 / 2$. The Thomas-FermiDirac equations represented here are based on the calculations of Metropolis and Reitz [1951], who determined the potential function $\psi$ as a function of nondimensional radius $x$ and gave the value $\psi\left(x_{0}\right)$ at the outer boundary of atoms $x_{0}$. Using these values, we get pressure $P$ and density $\rho$ of an element with atomic number $Z$ :

$$
\begin{aligned}
& P=\frac{Z^{2} e^{2}}{10 \pi \mu^{4}} {\left[\left(\frac{\psi\left(x_{n}\right)}{x_{0}}\right)^{1 / 2}+\epsilon\right]^{5} } \\
& \cdot {\left[1-\frac{5 \epsilon / \psi}{\left(\psi\left(x_{0}\right) / x_{0}\right)^{1 / 2}+\epsilon}\right] } \\
& \rho=3 Z M_{p} / 2 \pi\left(\mu x_{0}\right)^{3}
\end{aligned}
$$

where $e$ and $M_{p}$ are the electron charge and mass of proton, respectively, and $\mu$ and $G$ are constants given by

$$
\begin{aligned}
\mu & =0.46848 Z^{-1 / 3} \\
G & =0.21178 Z^{-2 / 3}
\end{aligned}
$$

$P$ - $\rho$ curves thus calculated for elements of atomic numbers $Z=14,29,33,49,61$, and 92 are given in Figure 8 . The $P_{-\rho}$ relations for the metals studied here are obtained by graphical interpolation.

Comparison of the $0^{\circ} \mathrm{K}$ isotherm obtained here for various metals with $P-\rho$ relations based on the Murnaghan-Birch and Thomas-Fermi-Dirac models are shown in Figures 1 to 7 . It can be seen that at lower pressures the Murnaghan-Birch

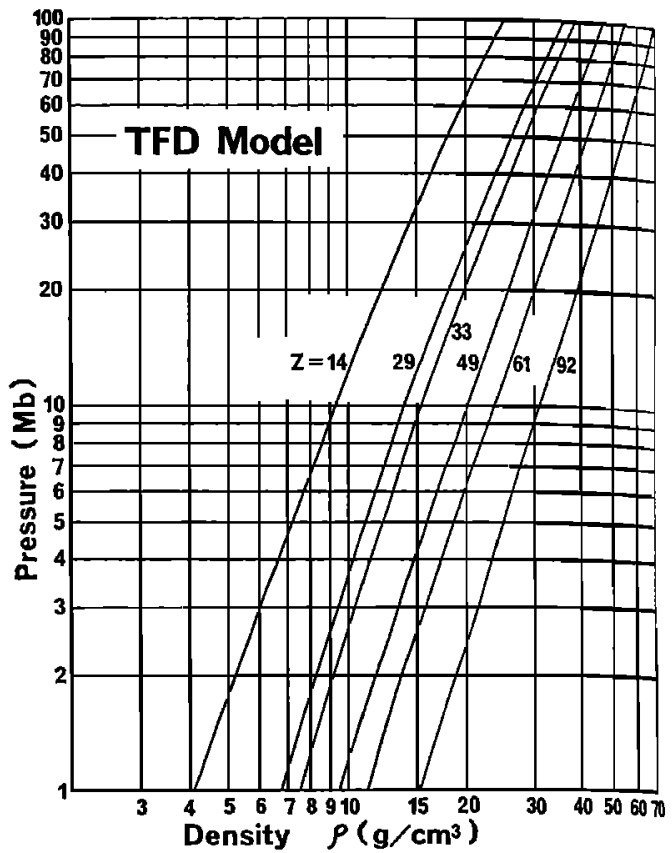

Fig. 8. Pressure-density relationships for the Thomas-Fermi-Dirac model of elements at $0^{\circ} \mathrm{K}$.

curves with $\xi=0 \approx-1 / 2$ fit the $P$ - $\rho$ relations of all the metals except titanium, for which the $P-\rho$ curve is approximated by a MurnaghanBirch curve with $\xi=1 / 2$. In other words, although the Murnaghan-Birch equation of state with $\xi=0$ does not approximate the equations of state of all the metals studied here as it does for a number of alkali metals [Birch, 1952], the equation of stite having a small second-order coefficient $\xi$ is quite appropriate for most of them. This second-order coefficient $\xi$ may vary from one metal to another, and it can be said that the equation of state of metals can essentially be determined by the two material constants, $k_{H o}$ and $\xi$. For some metals, such as Ag, $\mathrm{Cr}$, and $\mathrm{Fe}$, it can be seen that the $0^{\circ} \mathrm{K}$ isotherm would deviate from the Murnaghan-Birch curve and merge into the Thomas-Fermi-Dirac curve at pressures a little higher than $100 \mathrm{Mb}$.

In Figure 9, we compare calculated density and incompressibility of iron with those of the earth's core. Three density curves are calculated for temperatures $0^{\circ} \mathrm{K}, 3000^{\circ} \mathrm{K}$, and $6000^{\circ} \mathrm{K}$ by the method explained above. The temperature effect becomes small with increasing pressure. This figure shows that the density of the 


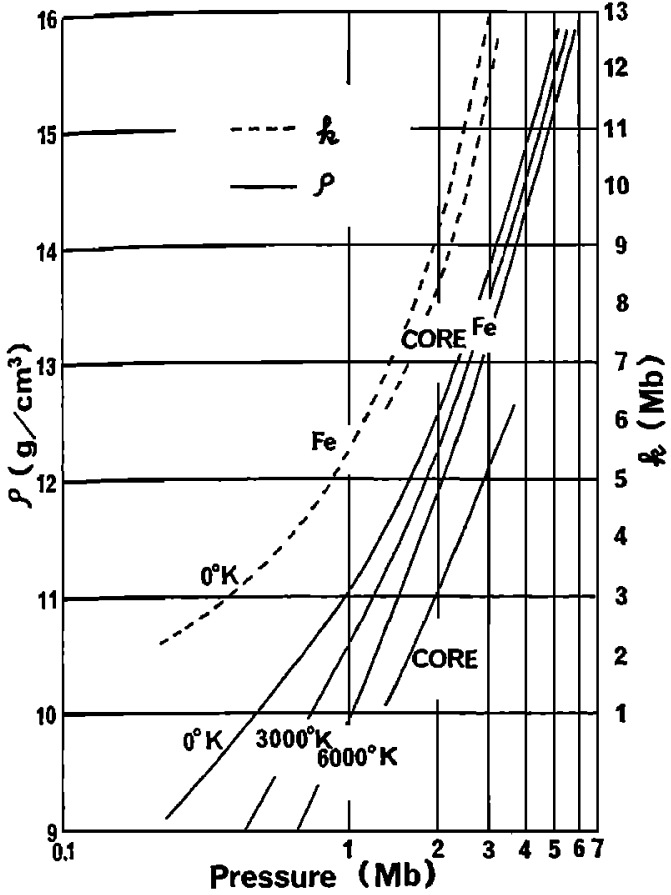

Fig. 9. Comparison of density and incompressibility curves for iron with those for the earth's core. Density distribution is based on Birch's solution $I$.

earth's core is 1 to $1.5 \mathrm{~g} / \mathrm{cm}^{3}$ smaller than that of iron at the pressure and temperature prevailing in the earth's core. The incompressibility curve of iron at $0^{\circ} \mathrm{K}$ is almost parallel to, and about $1 \mathrm{Mb}$ larger than, the incompressibility curve of the core. These results support the view [Knopoff and MacDonald, 1960] that the earth's core is composed of iron, silicon, and carbon.

The incompressibility-pressure hypothesis advanced by Bullen [1949] can be tested on the basis of the results obtained here. This hypothesis says that above a certain pressure the incompressibility $k$ is independent of the chemical composition and depends only on the pressure. In Table 5 are listed the values of the incompressibility of the metals at $0^{\circ} \mathrm{K}$ and at pressures of 0,1 , and $4 \mathrm{Mb}$. The range of the variation of the values of incompressibility among the metals studied here can be expressed by

$$
R=2\left(k_{\max }-k_{\min }\right) /\left(k_{\max }+k_{\min }\right)
$$

where $k_{\max }$ and $k_{\min }$ are the maximum and
TABLE 5. Incompressibilities of Metals (in Mb)

\begin{tabular}{|c|c|c|c|}
\hline \multirow[b]{2}{*}{ Metal } & \multicolumn{3}{|c|}{$P_{\mathrm{K}}, \mathrm{Mb}$} \\
\hline & 0 & 1 & 4 \\
\hline \multicolumn{4}{|c|}{ Al'tshuler et al. [1958] } \\
\hline Ag & 1.14 & 5.35 & 16.2 \\
\hline $\mathrm{Au}$ & 1.92 & 6.69 & 16.8 \\
\hline Cd & 0.61 & 4.36 & 13.9 \\
\hline $\mathrm{Cu}$ & 1.36 & 5.35 & 15.5 \\
\hline $\mathrm{Fe}$ & 1.14 & 5.52 & 16.8 \\
\hline $\mathrm{Pb}$ & 0.60 & 3.65 & 10.9 \\
\hline $\mathrm{Zn}$ & 0.73 & 4.44 & 13.8 \\
\hline \multicolumn{4}{|c|}{ McQueen and Marsh [1960] } \\
\hline Ag & 1.10 & 5.47 & 16.8 \\
\hline $\mathrm{Au}$ & 1.82 & 6.13 & 17.9 \\
\hline $\mathrm{Cd}$ & 0.52 & 5.00 & 16.3 \\
\hline Co & 1.99 & 5.65 & 14.8 \\
\hline Cr & 1.93 & 6.14 & 16.6 \\
\hline $\mathbf{C u}$ & 1.39 & 5.60 & 16.1 \\
\hline Mo & 2.71 & 6.14 & 14.6 \\
\hline $\mathrm{Ni}$ & 1.91 & 5.98 & 16.3 \\
\hline $\mathrm{Pb}$ & 0.47 & 4.29 & 13.9 \\
\hline Sn (gray) & 0.51 & 4.21 & 13.4 \\
\hline Sn (white) & 0.51 & 4.23 & 13.4 \\
\hline Th & 0.53 & 3.55 & 10.7 \\
\hline $\mathbf{T i}$ & 1.03 & 3.62 & 9.51 \\
\hline $\mathbf{T l}$ & 0.41 & 4.21 & 13.7 \\
\hline $\mathrm{V}$ & 1.59 & 4.77 & 12.4 \\
\hline W & 3.07 & 6.64 & 15.6 \\
\hline $\mathrm{Zn}_{\mathrm{n}}$ & 0.66 & 4.76 & 15.2 \\
\hline \multicolumn{4}{|c|}{$2\left(k_{\max }-k_{\min }\right)$} \\
\hline$k_{\text {roax }}$ & 1.53 & 0.61 & 0.61 \\
\hline
\end{tabular}

minimum values of the incompressibility. $R$ decreases very rapidly from 1.53 to 0.61 when the pressure increases from 0 to $1 \mathrm{Mb}$. However, $R$ does not change appreciably at pressures higher than $1 \mathrm{Mb}$. Consequently, it is expected that the incompressibility might differ from one material to another by at least $60 \%$ even at $4 \mathrm{Mb}$, which is approximately the pressure at the earth's center. The incompressibility-pressure hypothesis might not be true in a strict sense, but the important conclusion derived from this hypothesis, namely that a solid inner core accounts for the $10 \%$ increase in compressional wave velocity at the inner core boundary, might still be valid for the following reason. If the inner core is not solid, its incompressibility should be about $20 \%$ larger than that of the outer core. Since the incompressibility of the outer core is, as mentioned earlier, close to the incompressibility of iron, the incompressibility of the inner core should be about $20 \%$ larger than that of 
iron. As shown in Table 5, it is rather difficult to find a metal that satisfies this requirement, and this could make it impossible for the inner core to be liquid.

\section{Concluston}

The Mie-Grïneisen equation of state combined with the Slater or the Dugdale-MacDonald formula for the Grüneisen ratio can successfully be used for the interpretation of Hugoniot $P-v$ relation in terms of the equation of state.

The result for iron obtained by this method is in good agreement with the result given by Al'tshuler et al. in which the value of Grineisen's ratio at high pressure is experimentally determined. The use of either the Slater or the Dugdale-MacDonald formula does not make an appreciable difference in the final result. A comparison of the results with those obtained with the Murnaghan-Birch equation shows that the equation of state up to $10 \mathrm{Mb}$ for the metals studied here can essentially be determined by two constants which are characteristic of each material. The first constant is the incompressibility which varies from one material to another. The second constant $\xi$ takes a value between $-1 / 2$ and $1 / 2$ for many metals.

The TFD model gives densities that are too low even at $10 \mathrm{Mb}$, but an indication can be found in some metals that the $P$ - $\rho$ curve will be well approximated by the TFD model at pressures slightly higher than $100 \mathrm{Mb}$.

The density of the earth's core is definitely less than that of iron at $T=3000^{\circ} \mathrm{K}$, and even at $6000^{\circ} \mathrm{K}$.

The value of incompressibility at $0^{\circ} \mathrm{K}$ varies as much as $60 \%$ from one material to another, even at the pressure of $4 \mathrm{Mb}$. This does not support the incompressibility-pressure hypothesis, but a detailed inspection of the values of incompressibility at $4 \mathrm{Mb}$ might rule out the possibility that the inner core is liquid.

Acknowledgments. We express our sincere thanks to Professor J. Verhoogen, who read the manuscript and gave valuable suggestions. Some of the numerical calculations in the present paper were computed at the computation center of the University of California, Berkeley.

\section{REFGRENCES}

Alder, B. J., Physics experiments with strong pressure pulses, in Solids Under Pressure, edited by W. Paul and D. M. Warschauer, pp. 385-420, McGraw-Hill Book Company, New York, 1963. Al'tshuler, L. V., S. B. Kormer, M. I. Brazhnil, L. A. Vladimirov, M. P. Speranskaya, and A. I. Funtikov, The isentropic compressibility of aluminum, copper, lead, and iron at high pressures, Soviet Phys. JETP, English Transl., 11, 766-775, 1960.

Al'tshuler, L. V., K. K. Krupnikov, and M. I Brazhnik, Dynamic compressibility of metals under pressures from 400,000 to $4,000,000$ atmospheres, Soviet Phys. JETP, English Transl, $\$ 4$, 614-619, 1958.

Al'tshuler, L. V., K. K. Krupnikov, B. N. Ledenev, V. I. Zhuchikhin, and M. I. Brazhnik, Dynamic compressibility and equation of state of iron under high pressure, Soviet Phys. JETP, English Transl., 34, 606-614, 1958.

Birch, Francis, Elasticity and constitution of the earth's interior, J. Geophys. Res., 57, 227-286, 1952.

Bullen, K. E., Compressibility-pressure hypothesis and the earth's interior, Monthly Notices Roy. Astron. Soc., Geophys. Suppl., 5, 355-368, 1949.

Kittel, C., Introduction to Solid Slate Physics, John Wiley \& Sons, New York, 1956.

Knopoff, L., Equations of state of matter at moderately high pressures, in High Pressure Physics and Chemistry, vol. 1, edited by R. S. Bradley, pp. 227-263, Academic Press, New York, 1963.

Knopoff, L., and G. J. F. MacDonald, An equation of state for the core of the earth, Geophys. J., 3, 68-77, 1960.

McQueen, R. G., and S. P. Marsh, Equation of state for nineteen metallic elements from shockwave measurements to two megabars, $J$. Appl. Phys., 31, 1253-1269, 1960.

Metropolis, N., and J. R. Reitz, Solutions of the Fermi-Thomas-Dirac equation, J. Chem. Phys, 19, 555-573, 1951.

Rice, M. H., R. G. McQueen, and J. M. Walsh, Compression of solids by strong shock waves, Solid State Phys., 6, 1-63, 1958.

(Manuscript received January 25, 1966.) 